\title{
Pediatric neurosurgery telemedicine clinics: a model to provide care to geographically underserved areas of the United States and its territories
}

\author{
Hector E. James, MD \\ Lucy Gooding Pediatric Neurosurgery Center, Wolfson Children's Hospital/Baptist Health, and Division of Pediatric \\ Neurosurgery, University of Florida College of Medicine-Jacksonville, Jacksonville, Florida
}

OBJECTIVE The author describes the creation, structuring, and development of a pediatric neurosurgery telemedicine clinic (TMC) to provide telehealth across geographical, time, social, and cultural barriers.

METHODS In July 2009 the University of Florida (UF) Division of Pediatric Neurosurgery received a request from the Southeast Georgia Health District (Area 9-2) to provide a TMC to meet regional needs. The Children's Medical Services (CMS) of the State of Georgia installed telemedicine equipment and site-to-site connectivity. Audiovisual connectivity was performed in the UF Pediatric Neurosurgery office, maintaining privacy and HIPAA (Health Insurance Portability and Accountability Act) requirements. Administrative steps were taken with documentation of onsite training of the secretarial and nursing personnel of the CMS clinic. Patient preregistration and documentation were performed as required by the UF College of Medicine-Jacksonville. Monthly clinics are held with the CMS nursing personnel presenting the pertinent clinical history and findings to the pediatric neurosurgeon in the presence of the patient/parents. Physical findings and diagnostic studies are discussed, and management decisions are made.

RESULTS The first TMC was held in August 2011. A total of 40 TMC sessions have been held through January 2016, with a total of 43 patients seen: 13 patients once; 13 patients twice; 8 patients for 3 visits; 2 for 4 visits; 2 for 6 visits; 2 for 5 visits; 2 for 7 visits; and 1 patient has been seen 8 times.

CONCLUSIONS Pediatric patients in areas of the continental US and its territories with limited access to pediatric neurosurgery services could benefit from this model, if other pediatric neurosurgery centers provide telehealth services.

http://thejns.org/doi/abs/10.3171/2016.6.PEDS16202

KEY WORDS pediatric neurosurgery; telemedicine; geographically/socially underserved areas

$\mathrm{T}$ ELEMEDICINE, as defined by Darkins and Cary, ${ }^{3}$ is the use of advanced telecommunications technologies to exchange health information and provide health care services across geographical, time, social, and cultural barriers. In many areas of the continental United States and its territories, access to pediatric neurosurgery consultations and services is limited. This may be due in part to the limited number of pediatric neurological surgeons, clustering of neurosurgeons to medical centers, and the nonuniform geographical distribution of pediatric neurosurgery services. As a consequence, parents and families with an infant, child, or adolescent with neurosurgical needs may not be able to seek consultation and/or pediat- ric neurosurgery services in a timely fashion. In addition, they may be deprived of continuity of long-term care.

This article describes the structuring, development, initiation, and experience of a pediatric neurosurgery telemedicine clinic (TMC) providing health care to a geographically deprived region in the continental US. The purpose of this report is to communicate this information to pediatric neurosurgery services so that others may attempt to provide neurosurgical telehealth to underserved regions of the US and its territories.

\section{Methods}

In July 2009, communications were established be-

ABBREVIATIONS CMS = Children's Medical Services; HIPAA = Health Insurance Portability and Accountability Act; TMC $=$ telemedicine clinic; UF $=$ University of Florida SUBMITTED April 7, 2016. ACCEPTED June 21, 2016. 
tween the author (then the chief of the University of Florida [UF] Division of Pediatric Neurosurgery/Lucy Gooding Pediatric Neurosurgery Center at Wolfson Children's Hospital) and the State of Georgia Children's Medical Services (CMS), to provide pediatric neurosurgery TMCs. The patient site was to be located at the Waycross CMS Clinic, serving the Southeast Georgia Health District Area 9-2. Following exchange of pertinent administrative information and authorizations, an organizational scheme was developed. This consisted of, but was not limited to, an administrative matrix, electronic and connectivity matrix, and a patient care matrix.

\section{Administrative Matrix}

The purpose of this matrix was to care for mechanisms needed for patient identification, registration, referral sources, medical insurance, and documentation of health care information prior to patient contact. The following steps were to be taken prior to patient telemedicine contact, as per UF outpatient registration policies and procedures: patient registration forms were to be completed, signatures were to be obtained on consent forms as per HIPAA (Health Insurance Portability and Accountability Act) requirements, pertinent past and current medical information was to be forwarded to the pediatric neurosurgery office by facsimile, and neuroimaging studies were to be forwarded to the pediatric neurosurgery office on compact disc or by means of an equivalent method. The parents/caregivers were informed that they were required to accompany their child on the day of the clinic visit.

\section{Electronic and Connectivity Matrix}

The telemedicine systems were to be technically adequate to meet audiovisual needs, allowing for online communications between parties for history taking and physical examination. In addition, site-to-site connectivity was to be readily available, maintaining patient privacy and HIPAA requirements. Clinic visits were to take place in consulting rooms adequate to ensure patient privacy.

\section{Patient Care Matrix}

Allied health personnel for the clinic were to be educated on telemedicine patient encounters, common pediatric neurosurgical conditions, as well as follow-up needs. The telemedicine clinic (TMC) encounters were to be held face-to-face in the presence of the patient, parent/caregiver, CMS clinic nurse, and the consulting pediatric neurosurgeon. Documentation with pertinent coding information was to be completed for every patient encounter. A written consultation report was generated following the encounter and forwarded to the patient's referring physician as well as to the CMS TMC site. The consultations were then housed in the patient's medical record established with the UF Division of Pediatric Neurosurgery. At a subsequent date, when the electronic medical record (EMR) system was initiated, the documents were scanned into the UF EMR system (EPIC).

\section{Onsite Education}

On April 27, 2010, onsite training was performed at the
CMS Clinic in Waycross, Georgia. Training consisted of education on the administrative matrix elements by the office manager of the UF Division of Pediatric Neurosurgery to the secretarial personnel involved in the patient registration process in Waycross. The necessary documents for the registration process as required by the UF policies and procedures were distributed. The pediatric neurosurgeon provided the patient care matrix in the form of an overview session on clinical aspects of pediatric neurosurgery to the nursing personnel. Pertinent reference materials and parent education handouts on common pediatric neurological surgery conditions were distributed. Sign-in sheets were completed by the participants for the purpose of attendance documentation. The pediatric neurosurgeon subsequently created a written report for quality-improvement purposes.

\section{Additional Nursing Education}

In July 2010, the nursing personnel of the Waycross CMS Clinic visited the Lucy Gooding Pediatric Neurosurgery Center at Wolfson Children's Hospital in Jacksonville, Florida. They observed the evaluation and physical and neurological examination of new as well as follow-up patients and discussed clinical aspects of patient care. Emphasis was placed on the care of patients with multidisciplinary, long-term neurosurgical and rehabilitation needs. For the purpose of this paper we will refer to these patients as children with special health care needs.

\section{Medical License Requirements}

Through 2010, the pediatric neurosurgery faculty processed and completed the application process for obtaining a Georgia medical license, as required by the State of Georgia Medical Composite Board, to be able to provide medical care to patients in the State of Georgia.

\section{Electronic and Connectivity Installation}

In January 2011, the Southeast Georgia Health District completed the installation of the telehealth equipment in the Lucy Gooding Pediatric Neurosurgery Center TMC consultation room. A dedicated site-to-site circuit (circuit type: DS1, DS framing, clear channel) was connected by the telephone company and tested.

\section{Initial/Rehearsal Clinic}

In February 2011 a "rehearsal" clinic was performed. Consultations were scheduled for 3 patients along with their parents and/or caregivers. Preregistration procedures were completed and site-to-site connectivity was performed without difficulty. The patients and their parents/ caregivers were presented by the clinic nurses to the pediatric neurosurgeon. Documentation of the consultation followed, and medical coding and billing charges were generated successfully.

\section{Results \\ Patient Care}

Having concluded that the rehearsal TMC addressed administrative and medical issues, it was decided to pro- 
ceed in scheduling monthly half-day TMC sessions beginning in August 2011.

This report addresses the TMC activities through January 2016. A total of $40 \mathrm{TMC}$ sessions were held from August 2011 through January 2016. Of note: there were months sporadically during this period in which no patients were scheduled to be seen; therefore, TMC sessions were not held. A total of 43 patients (25 male and 18 female) have been evaluated through the TMC, with ages ranging from 4 months to 20 years. Thirteen patients were seen once; 13 patients twice; 8 patients for 3 visits; 2 for 4 visits; 2 for 6 visits; 2 for 5 visits; 2 for 7 visits; and 1 patient has been seen 8 times.

The clinical diagnoses for these patients are listed in Table 1 . The majority of the patients had hydrocephalus, craniosynostosis, or cranial deformities. Most patients were seen on a scheduled basis, referred by the local primary care physician and/or the CMS nurses of the Georgia Southeast Health District (Area 9-2 and neighboring areas). Some patients were referred to the TMC by the UF Division of Pediatric Neurosurgery after being treated in Jacksonville and having been identified as residents of the CMS District 9-2. Subsequent to the TMC visit, patients who required multidisciplinary clinic support, such as evaluations and/or periodic assessments by medical teams, were provided with appointments for the corresponding clinics in Jacksonville, including, but not limited to: the Spinal Defects Clinic, Spasticity Clinic, Pediatric Neurosciences Clinic, Neuro-Oncology Clinic, and Physical Medicine and Rehabilitation Clinic. Patients who required neurosurgical interventions had the operative procedures performed by the UF Division of Pediatric Neurosurgery at Wolfson Children's Hospital, a regional pediatric tertiary care center, which is a 1.5-hour drive from Waycross, Georgia. The timing of the procedures was determined by the patients' clinical status. No patients were seen because of an acute medical condition. Patients in need of acute or urgent care were referred to the emergency departments of local hospitals and not to CMS clinics. In turn, the emergency department physicians placed telephone consultations to the on-call pediatric neurosurgeon in Jacksonville.

\section{Nursing Aspects}

The CMS TMC nurses were in attendance during each TMC session. They are familiar with the patient/family medical and social conditions and were able to summarize pertinent clinical changes. They listened to the clinical questions posed by the neurosurgeon and, when instructed to do so, performed physical and neurological examinations so the neurosurgeon could hear and visualize them in real time. The nurses then noted the instructions in reference to studies, referrals, and follow-up visits. Following the TMC encounter, they completed referrals and study order forms that were requested. When the nurses ascertained the results, these were forwarded by mail or facsimile to the pediatric neurosurgeon. If questions arose between clinic sessions, the CMS nurses contacted the Lucy Gooding Pediatric Neurosurgery Center by email or telephone, requesting information or clarification of diagnostic studies and/or results.
TABLE 1. Clinical diagnoses of the 43 patients evaluated through the Lucy Gooding Pediatric Neurosurgery Center TMC as of January 2016

\begin{tabular}{lc}
\hline \multicolumn{1}{c}{ Diagnosis } & No. of Patients \\
\hline Hydrocephalus & 12 \\
\hline Craniosynostosis or cranial deformity & 12 \\
\hline Myelomeningocele & 8 \\
\hline Brain or spine tumor & 3 \\
\hline Head injury (follow-up) & 2 \\
\hline Syringomyelia & 2 \\
\hline Cranial arachnoid cyst & 1 \\
\hline Macrocrania & 1 \\
\hline Skull mass & 1 \\
\hline Spinal dermoid tract & 1 \\
\hline
\end{tabular}

\section{Administrative Aspects}

The CMS clinic administrative personnel obtained pertinent insurance and medical information from the patient/ parent prior to the scheduled clinic session. If the pertinent information was not completed, the session was rescheduled for a future appointment. Georgia CMS and Medicaid patients had their insurance verification completed by the CMS clinic personnel. If the patient had medical insurance other than CMS/Medicaid, the appropriate authorization was obtained prior to the clinic visit. Both medical insurance and clinical information were forwarded to the UF Division of Pediatric Neurosurgery by facsimile 1 week prior to the scheduled clinic date. This permitted the documentation to be verified by UF secretaries, as well as allowing them to prepare and/or update the patient's chart. On rare occasions, CMS scheduled appointments for patients whose medical insurance was not completed, although their medical eligibility had been verified; in these cases, Georgia CMS/Medicaid would subsequently authorize retroactive payment for services. On occasion, the CMS clinic nurses identified patients who they felt required a neurosurgical consultation, but Medicaid Managed Care would not authorize the visit. In those instances, the nurses requested that the neurosurgeon see the patient anyway for the purpose of determining the medical diagnosis. When the pediatric neurosurgeon completed the consultation and verified a neurosurgical diagnosis, the CMS procedures were put in place for "disenrollment" from Medicaid Managed Care and implementation of "straight" Medicaid coverage. Retroactive payment was then processed for those services.

Upon completion of the telemedicine consult and medical report, the medical billing code was attached. The medical code (CPT) employed is the same as a "face-toface" visit in the neurosurgery clinic, with an addition of a 2-digit "technical modifier." The charges are then submitted by the UF Coding and Billing Unit to Georgia Medicaid or the corresponding insurance carrier.

\section{Discussion}

Telemedicine has been defined as "the use of medical 
information exchanged from one site to another via electronic communications to improve a patient's health status." Therefore, this term is typically employed for the use of these technologies when a provider delivers direct patient health services. Telehealth, although often used interchangeably with telemedicine, is a broader term that includes telemedicine, as well other health-related services employing electronic information and communication, sharing information exchange, education, and other healthrelated services. ${ }^{1}$

Telemedicine technologies are tools that may be used to deliver the same or enhanced care that physicians currently provide, but over a distance. It is common for infants, children, and adolescents and their families living in suburban and urban communities to face significant disparities in access and time/distance barriers, which may be alleviated by the structured use of telemedicine services. ${ }^{19}$ This in turn can expand all aspects of patient- and family-centered health care.

Telemedicine has the potential to improve access to care, provide more patient- and family-centered care, increase efficiencies in medical practice, enhance local quality of health care, and address shortages in the clinical work force.${ }^{16}$ Telemedicine has the potential of linking primary and subspecialty care to maximize continuity of care, especially for children with special health care needs..$^{10,16}$ Telemedicine provides a venue for delivering subspecialty care to geographically deprived regions, in addition to reducing the burden of time away from work for parents/ caregivers, as well as the cost and time of travel. It is also a venue for providing ongoing education to physicians and nurses in remote locations to facilitate patient care at the primary medical home. ${ }^{16}$ In pediatrics, telemedicine is being employed in a variety of ways to deliver health services to children in primary care as well as subspecialty care. $4,5,7,8,11,12,17,18,22$ In addition to providing improved and timely access to care, telehealth has been demonstrated to reduce health care costs in pediatric primary and subspecialty care, as well as reducing redundancies in evaluative and diagnostic studies. ${ }^{9} 13-15,20,21,25$ Telehealth enhances both comfort and facility in managing specific medical subspecialty issues. ${ }^{2,6,24}$ Clinical programs have demonstrated an increase in the number of patients cared for as well as the overall quality of care provided to patients with specific medical conditions. ${ }^{2,23}$

Our telemedicine clinic experience with CMS Georgia has provided a unique venue for patient care and education. The CMS nurses have expressed their satisfaction not only for the experience, but also on behalf of the parents/ caregivers of children with a neurosurgical condition. In addition, the nurses have indicated that with each session they have a forum of continuing medical education that they would otherwise never experience.

In the United States, the practice of medicine is regulated by each state through the state medical boards and/ or equivalent agencies. To practice telemedicine in a state, the physician must practice under the licensing agency of the state in which the patient resides. In the process, the physician indicates he or she is providing medical care within the scope of their specialty as it should be documented in the application to the medical board. Under these circumstances, the physician is usually covered by their corresponding malpractice insurance. It should be pointed out that there are administrative variations in the medical malpractice industry, and this should be clarified by the physician prior to initiating any telemedicine endeavor.

In the United States, timely access to pediatric neurosurgery consultations and continuity of care may be impacted by the limited number of pediatric neurosurgeons, their geographical distribution, the "clustering" of neurological surgeons to centers with the appropriate resources, and subspecialty support. Aside from the issue of limited access in rural areas when compared with suburban areas in the continental US, there is also limited access to children with neurosurgical needs in the US territories.

\section{Conclusions}

The successful establishment of a pediatric neurosurgery TMC providing services across state lines to a geographically deprived area has been accomplished and was performed by collaboration between state and university administrations, interdisciplinary communications, and education, centered around the needs of the children and families.

The intention for this paper was to present a potential model of a pediatric neurosurgery TMC program in an attempt to raise interest in the pediatric neurosurgery leadership and its organizations, as well as other pediatric neurosurgery centers, regarding the provision of telehealth to those communities in the continental US and its territories that are geographically and socially deprived.

\section{Acknowledgments}

I wish to acknowledge the dedicated secretarial support in the preparation and electronic submission of this report provided by Jennifer Santarone.

Additionally, I wish to acknowledge that this work has been supported in part by the Lucy B. Gooding Charitable Foundation Trust and the Baptist Health Foundation of Northeast Florida, both of which are not-for-profit organizations.

The telemedicine equipment, connectivity, and ongoing support for the clinics are provided by the Children's Medical Services of the State of Georgia.

\section{References}

1. American Telemedicine Association: What is Telemedicine? (http://www.americantelemed.org/about-telemedicine/what-istelemedicine\#.VSKdNfnF98E) [Accessed July 15, 2016]

2. Arora S, Thornton K, Murata G, Deming P, Kalishman S, Dion D, et al: Outcomes of treatment for hepatitis C virus infection by primary care providers. N Engl J Med 364:2199-2207, 2011

3. Darkins AW, Cary MA (eds): Telemedicine and Telehealth: Principles, Policies, Performance and Pitfalls. New York: Springer, 2000

4. Desai S, Williams ML, Smith AC: Teleconsultation from a secondary hospital for paediatric emergencies occurring at rural hospitals in Queensland. J Telemed Telecare 19:405410, 2013

5. Dharmar M, Romano PS, Kuppermann N, Nesbitt TS, Cole SL, Andrada ER, et al: Impact of critical care telemedicine 
consultations on children in rural emergency departments. Crit Care Med 41:2388-2395, 2013

6. Gray JE, Safran C, Davis RB, Pompilio-Weitzner G, Stewart JE, Zaccagnini L, et al: Baby CareLink: using the internet and telemedicine to improve care for high-risk infants. Pediatrics 106:1318-1324, 2000

7. Hall-Barrow J, Hall RW, Burke BL Jr: Telemedicine and neonatal regionalization of care-ensuring that the right baby gets to the right nursery. Pediatr Ann 38:557-561, 2009

8. Herendeen NE, Schaefer GB: Practical applications of telemedicine for pediatricians. Pediatr Ann 38:567-569, 2009

9. Jackson KM, Scott KE, Graff Zivin J, Bateman DA, Flynn JT, Keenan JD, et al: Cost-utility analysis of telemedicine and ophthalmoscopy for retinopathy of prematurity management. Arch Ophthalmol 126:493-499, 2008

10. Karp WB, Grigsby RK, McSwiggan-Hardin M, PursleyCrotteau S, Adams LN, Bell W, et al: Use of telemedicine for children with special health care needs. Pediatrics 105:843847,2000

11. Kim EW, Teague-Ross TJ, Greenfield WW, Keith Williams D, Kuo D, Hall RW: Telemedicine collaboration improves perinatal regionalization and lowers statewide infant mortality. J Perinatol 33:725-730, 2013

12. Labarbera JM, Ellenby MS, Bouressa P, Burrell J, Flori HR, Marcin JP: The impact of telemedicine intensivist support and a pediatric hospitalist program on a community hospital. Telemed J E Health 19:760-766, 2013

13. Mahnke CB, Jordan CP, Bergvall E, Person DA, Pinsker JE: The Pacific Asynchronous TeleHealth (PATH) system: review of 1,000 pediatric teleconsultations. Telemed J E Health 17:35-39, 2011

14. Marcin JP, Ellis J, Mawis R, Nagrampa E, Nesbitt TS, Dimand RJ: Using telemedicine to provide pediatric subspecialty care to children with special health care needs in an underserved rural community. Pediatrics 113:1-6, 2004

15. Marcin JP, Nesbitt TS, Struve S, Traugott C, Dimand RJ: Financial benefits of a pediatric intensive care unitbased telemedicine program to a rural adult intensive care unit: impact of keeping acutely ill and injured children in their local community. Telemed J E Health 10 (Suppl 2):S-1-S-5, 2004

16. Marcin JP, Rimsza ME, Moskowitz WB: The use of telemedicine to address access and physician workforce shortages. Pediatrics 136:202-209, 2015

17. McConnochie KM, Wood NE, Kitzman HJ, Herendeen NE, Roy J, Roghmann KJ: Telemedicine reduces absence resulting from illness in urban child care: evaluation of an innovation. Pediatrics 115:1273-1282, 2005

18. Randolph GD, Pathman DE: Trends in the rural-urban distribution of general pediatricians. Pediatrics 107:E18, 2001

19. Skinner AC, Slifkin RT: Rural/urban differences in barriers to and burden of care for children with special health care needs. J Rural Health 23:150-157, 2007

20. Smith AC, Scuffham P, Wootton R: The costs and potential savings of a novel telepaediatric service in Queensland. BMC Health Serv Res 7:35, 2007

21. Spaulding R, Belz N, DeLurgio S, Williams AR: Cost savings of telemedicine utilization for child psychiatry in a rural Kansas community. Telemed J E Health 16:867-871, 2010

22. Spooner SA, Gotlieb EM: Telemedicine: pediatric applications. Pediatrics 113:e639-e643, 2004

23. Wielawski IM: Reach out: physicians' initiative to expand care to underserved Americans, in Isaacs SL, Knickman JK (eds): To Improve Health and Health Care, 1997: The Robert Wood Johnson Foundation Anthology. San Francisco: Jossey-Bass, 1997, pp 1-18

24. Wootton R: Twenty years of telemedicine in chronic disease management - an evidence synthesis. J Telemed Telecare 18:211-220, 2012

25. Yang NH, Dharmar M, Kuppermann N, Romano PS, Nesbitt TS, Hojman NM, et al: Appropriateness of disposition following telemedicine consultations in rural emergency departments. Pediatr Crit Care Med 16:e59-e64, 2015

\section{Disclosures}

The author reports no conflict of interest concerning the materials or methods used in this study or the findings specified in this paper.

\section{Correspondence}

Hector E. James, Lucy Gooding Pediatric Neurosurgery, 836 Prudential Dr., Ste. 1205 Pavilion Bldg., Jacksonville, FL 32207. email: hector.james@jax.ufl.edu. 